POS $\quad$ PROCEEDINGS

\title{
Dilepton measurements with CERES
}

Ana Marín for the CERES Collaboration: D. Adamováa ${ }^{a}$ G. Agakichiev ${ }^{b}$,

A. Andronic ${ }^{c}$, D. Antończyk ${ }^{d}$, H. Appelshäuser ${ }^{d}$, V. Belaga ${ }^{b}$, J. Bielčíkováe, ${ }^{,}$,

P. Braun-Munzinger ${ }^{c}$, O. Busch ${ }^{c}$, A. Cherling, S. Damjanovićf ${ }^{f}$ T. Dietel ${ }^{\mathrm{h}}$, L. Dietrich ${ }^{\mathrm{f}}$,

A. Drees ${ }^{\mathrm{i}}$, S. I. Esumi ${ }^{\mathrm{f}}$, K. Filimonov ${ }^{\mathrm{f}}$, K. Fomenko ${ }^{\mathrm{b}}$, Z. Fraenkel ${ }^{\mathrm{g}}$, C. Garabatos ${ }^{\mathrm{c}}$,

P. Glässel ${ }^{\mathrm{f}}$, G. Hering ${ }^{\mathrm{c}}$, J. Holeczek ${ }^{\mathrm{c}}$, M. Kalisky ${ }^{\mathrm{c}}$, S. Kniege ${ }^{\mathrm{d}}$, V. Kushpila,

W. Ludolphs ${ }^{f}$, A. Maas ${ }^{c}$, A. Marín ${ }^{c}$, J. Miloševićf ${ }^{f}$ D. Miśkowiec ${ }^{c}$, R. Ortega ${ }^{f}$,

Y. Panebrattsev ${ }^{\mathrm{b}}$, O. Petchenova ${ }^{\mathrm{b}}$, V. Petráček ${ }^{\mathrm{f}}$, M. Płoskońd $^{\mathrm{d}}$, S. Radomski ${ }^{\mathrm{f}}$, J. Rak $^{\mathrm{c}}$,

I. Ravinovich ${ }^{g}$, P. Rehak ${ }^{j}$, H. Sako ${ }^{c}$, W. Schmitz ${ }^{\mathrm{f}}$, S. Schuchmann ${ }^{\mathrm{d}}$, S. Sedykh ${ }^{\mathrm{c}}$,

S. Shimansky ${ }^{b}$, R. Soualah ${ }^{\mathrm{f}}$, J. Stachel ${ }^{\mathrm{f}}$, M. Šumbera ${ }^{\mathrm{a}}$, H. Tilsner $^{\mathrm{f}}$, I. Tserruya ${ }^{\mathrm{g}}$,

G. Tsiledakis ${ }^{c}$, J. P. Wessels ${ }^{\mathrm{h}}$, T. Wienold ${ }^{\mathrm{f}}$, J.P. Wurm ${ }^{\mathrm{e}}$, S. Yurevich ${ }^{\mathrm{c}}$, V. Yurevich $^{\mathrm{b}}$

${ }^{a}$ NPI ASCR, ̌̌ež, Czech Republic

b JINR Dubna, Russia

${ }^{\mathrm{c}}$ GSI Darmstadt, Germany

${ }^{\mathrm{d}}$ Frankfurt University, Germany

${ }^{\mathrm{e}} M P I$, Heidelberg, Germany

${ }^{\mathrm{f}}$ Heidelberg University, Germany

${ }^{\mathrm{g}}$ Weizmann Institute, Rehovot, Israel

${ }^{\mathrm{h}}$ Münster University, Germany

${ }^{i} S U N Y$ Stony Brook, U.S.A.

${ }^{\mathrm{j}}$ BNL, Upton, U.S.A.

${ }^{\mathrm{k}}$ CERN, Geneva, Switzerland

E-mail: a.marinegsi.de

\begin{abstract}
We report on dilepton measurements for central $\mathrm{Pb}$ on $\mathrm{Au}$ collisions at the top CERN SPS energy with the upgraded CERES experiment. The dilepton mass spectrum of 2000 data with improved mass resolution shows an enhancement over the expectation from hadron decays that is well described by a model including a strong broadening of the $\rho$ spectral function. The measured excess yield excludes the dropping mass scenario.

We also report on the $\phi$ meson measured simultaneously both in the $K^{+} K^{-}$and in the dilepton decay channel for the first time in high energy heavy-ion collisions. An excellent agreement is found between the rapidity densities and the shape of the measured tranverse momentum spectrum. The data rule out a possible enhancement of the $\phi$ yield in the leptonic over hadronic channel by a factor larger than 1.6 at $95 \%$ CL. CERES results are in agreement with NA49 results.
\end{abstract}

Critical Point and Onset of Deconfinement - 4th International Workshop

July 9 - 13, 2007

Darmstadt, Germany 


\section{Introduction}

The main goal of ultra-relativistic heavy-ion collisions is the study of very hot and dense nuclear matter. This kind of matter is believed to have existed shortly after the Big Bang. Lattice QCD calculations [1] have predicted a transition from ordinary hadronic matter to a plasma of quarks and gluons at high energy densities. At the same time Chiral symmetry is restored. Dileptons that carry information of the entire fireball evolution are very suitable probes because of their negligible final state interactions.

Medium modifications of the $\phi$ meson properties (mass and width) or of the kaons that might result in a change of the branching fraction of $\phi$ decaying to $\mathrm{K}^{+} \mathrm{K}^{-}$or to $e^{+} e^{-}$when $\phi$ decays in medium, may be related to the expected chiral phase transition [2]. However as the $\phi$ lifetime ( $44 \mathrm{fm} / \mathrm{c}$ ) is longer than the expected lifetime of the coupled collision system, only a fraction may decay in the hot fireball. Final state interactions of kaons from $\phi$ meson decays may lower the measured branching ratio into the kaon channel [3]. Indeed, the NA50 experiment measuring the $\phi$ meson in the $\mu^{+} \mu^{-}$decay channel and NA49 measuring in the $\mathrm{K}^{+} \mathrm{K}^{-}$channel, obtain yields that differ by factors between 2 and 4 in the common $m_{t}$ range [4]. Further, the $m_{t}$ spectra exhibit a different inverse slope parameter, $305 \pm 15 \mathrm{MeV}$ in NA49 and $218 \pm 6 \mathrm{MeV}$ in NA50, fitted in their $m_{t}$ acceptance regions.

The CERES experiment at the CERN SPS is an experiment dedicated to the study of low mass dilepton pairs. CERES has measured an enhanced dilepton production in the invariant mass region $m_{e^{+} e^{-}}>0.2 \mathrm{GeV} / \mathrm{c}^{2}$ in $\mathrm{S}+\mathrm{Au}$ at $200 \mathrm{AGeV}$ [5] and in $\mathrm{Pb}+\mathrm{Au}$ at $158 \mathrm{AGeV}$ [6]. The enhancement is absent in p-induced reactions [7]. Pion annihilation has been taken into account as an additional mechanism for $\mathrm{e}^{+} \mathrm{e}^{-}$production but the experimental spectra cannot be explained without introducing medium modifications of vector mesons, particularly of the $\rho$. The two main theoretical choices for this modification are the dropping mass scenario [8] and the broadening scenario [9], but the experimental precision did not allow to distinguish between them.

In order to further investigate the enhancement and possibly discriminate between the different theoretical approaches, the CERES spectrometer was upgraded during 1998 by the addition of a Time Projection Chamber (TPC) with radial electric drift field [10,11, 12, 13, 14, 15] which improves the mass resolution and the electron identification. In order to investigate the role of baryons, during 1999, CERES took data with the TPC (although with partial readout) at a bombarding energy of $40 \mathrm{AGeV}$ [16] where the baryon density is approximately $30 \%$ larger than at $158 \mathrm{AGeV}$ [17]. During the year 2000 CERES took a large data sample consisting of $30 \cdot 10^{6}$ and $3 \cdot 10^{6}$ events of $\mathrm{Pb}$ on Au collisions at $158 \mathrm{AGeV}$ triggered on the $7 \%$ and $20 \%$ most central collisions [14], respectively. Moreover, the upgrade of the CERES experiment makes possible for the first time in high energy heavy-ion collisions to study simultaneously the leptonic and the charged kaon decay modes of the $\phi$ meson at the SPS, thus shedding light onto the $\phi$ puzzle.

In this paper, we present an overview of the most relevant dilepton results of the CERES experiment since the upgrade with the TPC. All these results have been previously published in $[16,18,19]$. 


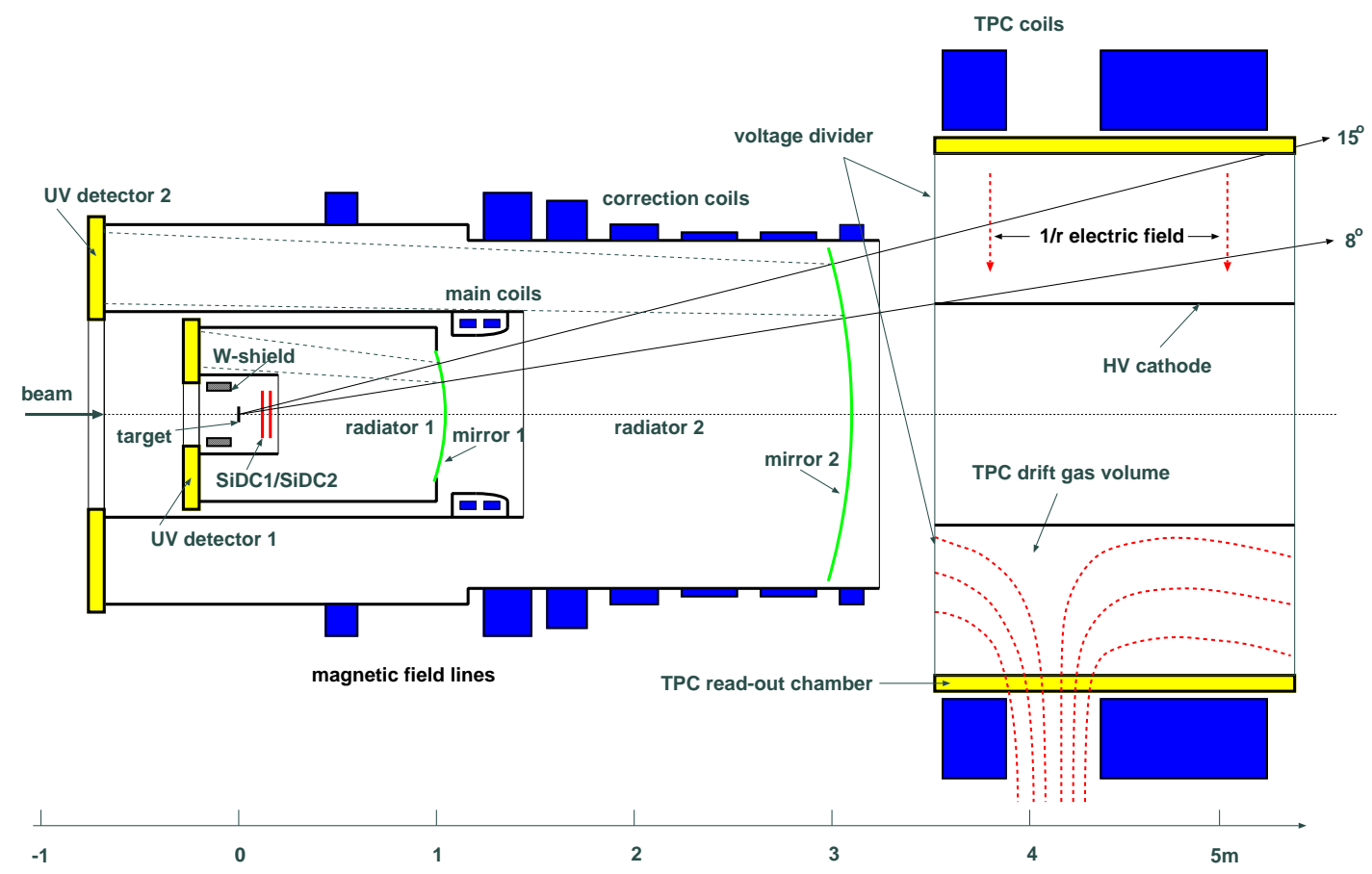

Figure 1: Cross section through the upgraded setup of the CERES spectrometer. The apparatus has a cylindrical symmetry.

\section{Experimental setup}

The CERES experiment (Fig. 1) is optimized to measure low mass electron pairs close to mid-rapidity $(2.1<\eta<2.6)$ with full azimuthal coverage. A vertex telescope, composed of two Silicon Drift Detectors (SDD) positioned at $10.4 \mathrm{~cm}$ and $14.3 \mathrm{~cm}$ downstream of a segmented $\mathrm{Au}$ target, provides a precise vertex reconstruction, angle measurement for charged particles and rejection of close pairs from $\gamma$ conversions and $\pi^{0}$ Dalitz decays. Two Ring Imaging CHerenkov (RICH) detectors, operated at a high threshold $\left(\gamma_{t h}=32\right)$, are used for electron identification in a large hadronic background. In the configuration with the TPC, the magnetic field between the two RICH detectors is switched off, allowing to use them in a combined mode resulting in an increased electron efficiency. The new radial-drift TPC, positioned downstream of the original spectrometer, has an active length of $2 \mathrm{~m}$ and a diameter of $2.6 \mathrm{~m}$. A gas mixture of $\mathrm{Ne}(80 \%)$ and $\mathrm{CO}_{2}(20 \%)$ is used. It is operated inside a magnetic field (indicated by the dashed field lines inside the TPC in Fig. 1) with a maximal radial component of $0.5 \mathrm{~T}$ and provides up to 20 space points for each charged particle track. This is sufficient for the momentum determination with a resolution $\Delta p / p \sim\left((2 \%)^{2}+(1 \% \cdot p(\mathrm{GeV} / \mathrm{c}))^{2}\right)^{1 / 2}$ and for additional electron identification by using the $\mathrm{d} E / \mathrm{d} x$ signal in the TPC.

\section{Di-electron analysis}

Charged particles from the target are reconstructed by matching track segments in the SDD and in the TPC using a momentum-dependent matching window. Tracks in the TPC are required 
to contain more than 12 hits out of a maximum 20 possible, to ensure good momentum resolution. Cherenkov rings with asymptotic radii are identified in the RICH using a Hough transformation (see sect. 3.2.4 in ref. [6]). The TPC electron selection is done based on the $\mathrm{d} E / \mathrm{d} x$ signal and its resolution. To select electrons among all charged hadrons both a TPC $\mathrm{d} E / \mathrm{dx}$ signal in the electron region and a matching to a RICH ring are required. The combined pion rejection factor varies from $4 \times 10^{4}$ to $1.8 \times 10^{4}$ for momenta between $1 \mathrm{GeV} / \mathrm{c}$ and $2.5 \mathrm{GeV} / \mathrm{c}$ for total electron efficiencies of $68 \%$ and $66 \%$ [20], respectively, with the quality cuts applied in the analysis [21, 20, 22].

The main difficulties of the electron analysis are the low probability of electromagnetic decays and the large amount of combinatorial background from $\gamma$ conversions and Dalitz decays. Very good electron identification is not enough. Electron pairs from $\gamma$ conversions and $\pi^{0}$ Dalitz decays, characterized by their small opening angle and low momentum, need to be identified by their topology and removed from the sample in order to reduce the combinatorial background. As the detectors have a finite two-track resolution the most effective way of rejecting conversions in the target and close Dalitz pairs is by rejecting tracks with an energy loss signal significatively larger than the minimum ionizing energy loss signal in both SDD's where all hit amplitudes have been resummed around each SDD track segment. Low-amplitude tails in the SDD dE/dx distribution are also removed. Late conversions (mostly in SDD1) are removed by a cut in the distance to another TPC track of opposite sign and with electron $\mathrm{d} E / \mathrm{dx}$. To reduce $\pi^{0}$ Dalitz decay contributions only electron tracks with no opposite charge electron track within $35 \mathrm{mrad}$ are taken for analysis. Finally, only electron tracks in the geometrical acceptance $0.141 \mathrm{rad}<\theta<0.243 \mathrm{rad}$ and with a $p_{t}>0.2 \mathrm{GeV} / \mathrm{c}$ are selected.

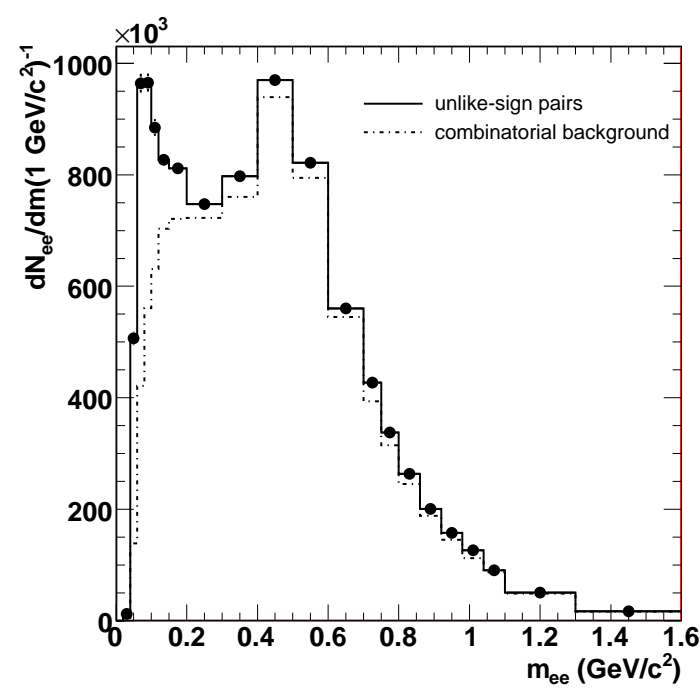

Figure 2: Invariant-mass distribution of unlike-sign pairs (histogram), and mixed-event background (dashed line) normalized to like-sign pairs background after full rejection and corrected for pair reconstruction efficiency event by event.

The invariant-mass distributions of unlike-sign pairs and of the combinatorial background after full rejection and corrected for pair reconstruction efficiency event by event are shown in Fig. 2. 

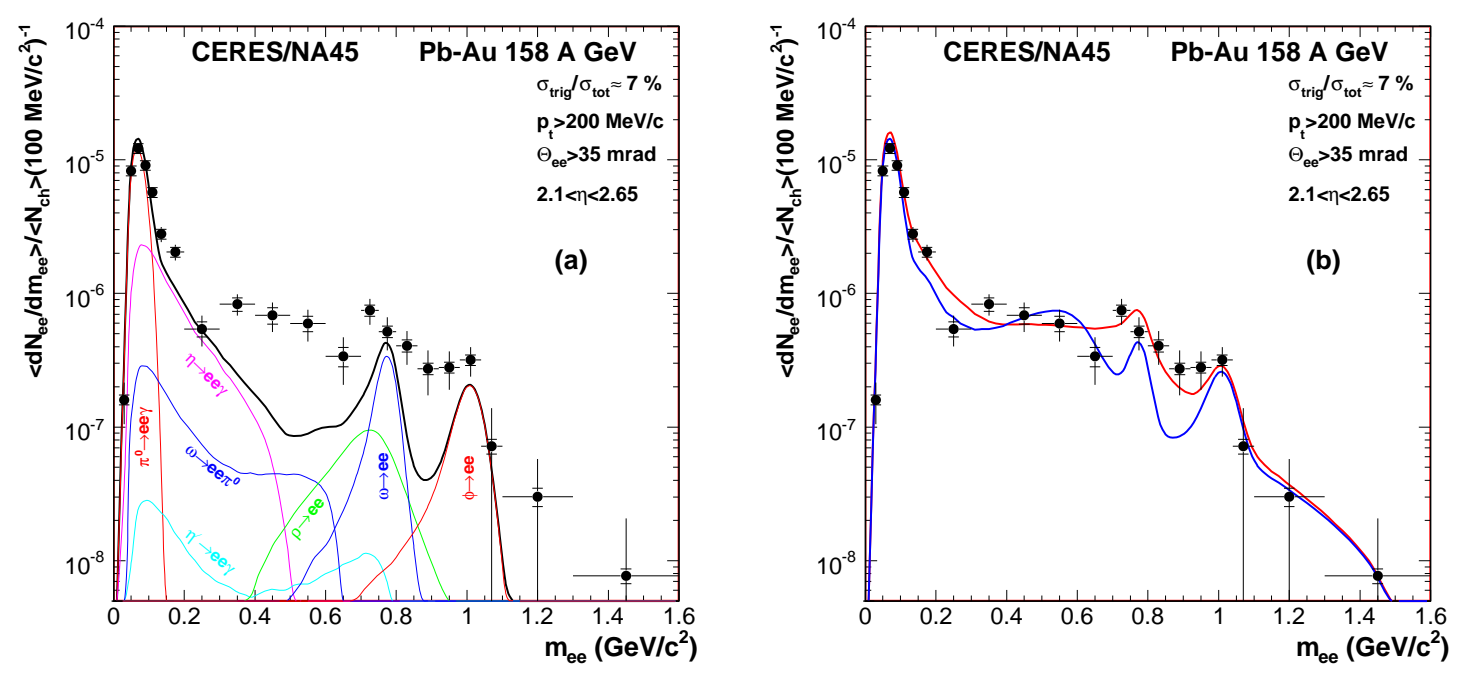

Figure 3: (a) Invariant-mass spectrum of $e^{+} e^{-}$-pairs compared to the expectation from the hadron decay cocktail. (b) The expectations from model calculations assuming a dropping of the $\rho$ meson mass (blue) or a spread of the $\rho$ width in the medium (red) are also shown.

In order to reduce the statistical errors an unlike-sign combinatorial background using the mixedevent technique has been evaluated. The mixed-event background is normalized to the like-sign pair background in the mass region $\mathrm{m}_{e^{+} e^{-}}>0.2 \mathrm{GeV} / \mathrm{c}^{2}$. For masses below $0.2 \mathrm{GeV} / \mathrm{c}^{2}$, the likesign background is used. The physics signal is obtained by subtracting the like-sign pairs or the mixed event background from the unlike-sign pairs.

\section{Low-mass $\mathrm{e}^{+} \mathrm{e}^{-}$results}

The $\mathrm{e}^{+} \mathrm{e}^{-}$invariant-mass spectrum corrected event-by-event for the pair reconstruction efficiency and normalized to the number of events and to the average charged particle multiplicity $<N_{c h}>$ in the acceptance [14] is shown in Fig. 3(a) [18] compared to the expectations from the hadronic decay cocktail. The hadron decay cocktail [23] has been folded with the experimental momentum resolution and energy loss due to bremsstrahlung. Acceptance, opening-angle, and transverse-momentum cuts are applied. An excess of pairs for $\mathrm{m}_{e^{+} e^{-}}>0.2 \mathrm{GeV} / c^{2}$ is clearly visible. The number of pairs in the Dalitz region $\left(\mathrm{m}_{e^{+} e^{-}}<0.2 \mathrm{GeV} / \mathrm{c}^{2}\right)$ is $6114 \pm 176$ with a signal to background ratio $(\mathrm{S} / \mathrm{B})$ of $1 / 2$. The number of open pairs $\left(\mathrm{m}_{e^{+} e^{-}}>0.2 \mathrm{GeV} / \mathrm{c}^{2}\right)$ is $3115 \pm 376$ with a $\mathrm{S} / \mathrm{B}$ of $1 / 22$. The enhancement factor for $0.2 \mathrm{GeV} / \mathrm{c}^{2}<\mathrm{m}_{e^{+} e^{-}}<1.1 \mathrm{GeV} / \mathrm{c}^{2}$ compared to the hadron decay cocktail is $2.56 \pm 0.22$ (stat) \pm 0.31 (syst) \pm 0.83 (decays) ${ }^{1}$. The total data systematic uncertainty of $12 \%$ includes the combinatorial background subtraction and the electron efficiency correction. The systematic error of the charged particle multiplicity determination of $12 \%$ has been included on the systematic error of the cocktail.

\footnotetext{
${ }^{1}$ The change on the branching ratio of the proccess $\omega \rightarrow \pi^{0} e^{+} e^{-}$and the improvement in its error in PDG 2006 have not been considered yet.
} 


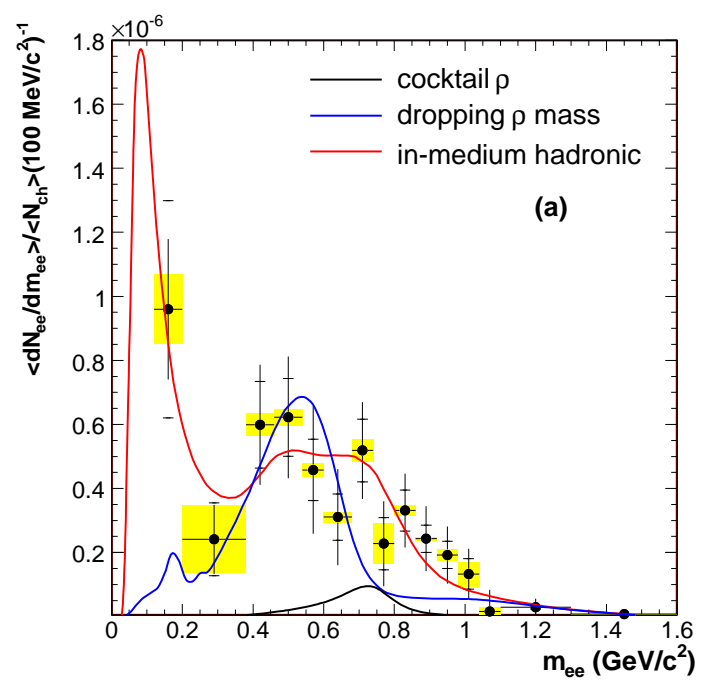

Figure 4: (a) Dilepton yield after subtraction of the hadronic cocktail (without the $\rho$ ). The systematic errors of the data (horizontal ticks) and the systematic uncertainty of the subtracted cocktail (shaded boxes) are also shown. The data are compared to expectations of the models assuming a shift of the in-medium $\rho$ mass (blue) and a broadening scenario (red).

The experimental results are compared to theoretical models 3(b) based on hadronic decays and $\pi^{+} \pi^{-}$annihilation. The $\rho$-propagator is treated [24] in 3 ways: vacuum $\rho$ (not shown), modifications following Brown-Rho scaling [8], and modifications via $\rho$-hadron interactions [9]. In the region between the $\omega$ and the $\phi$, measured now with better resolution, the data favor the many-body approach over Brown-Rho scaling.

\section{The in-medium $\rho$}

In order to further investigate the excess dilepton yield the contribution from the hadronic cocktail (except the $\rho$ ) is subtracted from the data and from the calculations (Fig. 4a). No rescaling of theory to data is used. It can be observed that the contribution of the cocktail $\rho$ is totally negligible. The in-medium $\rho$ contribution dominates by more than one order of magnitude (enhancement factor of $16.4 \pm 1.8$ for $0.12 \mathrm{GeV} / \mathrm{c}^{2}<\mathrm{m}_{e^{+} e^{-}}<1.1 \mathrm{GeV} / \mathrm{c}^{2}$ ) in central $\mathrm{Pb}$ on Au collisions. The shape and the yield are well described by the in-medium hadronic contribution. A detailed study of the spectrum width including systematic and statistical errors excludes the dropping $\rho$ mass scenario [18].

\section{The $\phi$ meson}

The $\phi$ meson yield in the $\mathrm{e}^{+} \mathrm{e}^{-}$channel is determined by integrating the invariant mass spectra in the mass region between 0.9 and $1.1 \mathrm{GeV} / \mathrm{c}^{2}$ in three transverse momentum bins [19]. The integrated yield in the mass range between 0.9 and $1.1 \mathrm{GeV} / \mathrm{c}^{2}$ is $229 \pm 53$ with a signal to background 


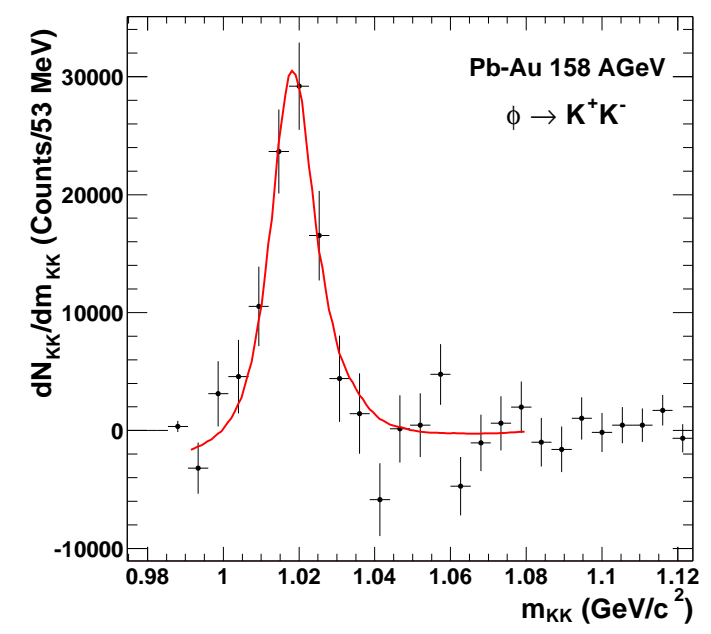

Figure 5: Invariant-mass spectra of $K^{+} K^{-}$pairs after background subtraction for the $\left(p_{t}^{\phi}, y^{\phi}\right)$ bin $1.5 \mathrm{GeV} / \mathrm{c}$ $<p_{t}^{\phi}<1.75 \mathrm{GeV} / \mathrm{c}$ and $2.2<y^{\phi}<2.4$.

ratio of $1 / 12$ and needs to be corrected for acceptance, reconstruction efficiency and physics background under the $\phi$ peak. The $\rho$ meson could extend into this mass range if its spectral function is modified in the medium. Dileptons from the QGP phase also contribute to the physics background in this mass range. The sum of these two contributions is estimated to be $35 \%$ of the total yield in this mass region by inspecting theoretical models that include in-medium spreading of the $\rho$ width due to $2 \pi$ processes and the dilepton yield from the QGP phase [9]. The measured $\phi$ yield has been scaled by this factor to correct for the physics background. The charm contribution that is smaller than 3\% [26] has been neglected.

As mentioned before, CERES can also study the $\phi$ meson in the charged kaon $\left(\mathrm{K}^{+} \mathrm{K}^{-}\right)$decay mode. In order to do so, all charged particles get assigned the kaon mass (no particle identification is used). Only a conservative upper cut in the $\mathrm{d} E / \mathrm{d} x$ signal (corresponding to $90 \%$ of the Fermi plateau value) for momenta between $1.25 \mathrm{GeV} / \mathrm{c}$ and $4 \mathrm{GeV} / \mathrm{c}$, suppressing $83 \%$ of the electrons, is applied to enhance the kaon content of the sample. Tracks in the geometrical acceptance $0.13 \mathrm{rad}$ $<\theta<0.24 \mathrm{rad}$ with a transverse momentum $p_{t}$ larger than $0.25 \mathrm{GeV} / \mathrm{c}$ are selected. To reduce the contamination from other particle species, cuts in the Podolanski-Armenteros parameter [27] and in the opening angle between the kaons are applied. The $\phi$ meson in the kaon decay mode is studied in the rapidity interval $2.0<y^{\phi}<2.4$ for $p_{t}^{\phi}>0.75 \mathrm{GeV} / \mathrm{c}$.

The invariant mass distributions of $\mathrm{K}^{+} \mathrm{K}^{-}$pairs were accumulated in $\left(p_{t}^{\phi}, y^{\phi}\right)$ bins to obtain the $\phi$ transverse momentum spectrum. The combinatorial background invariant mass distributions are calculated using the mixed-event technique for each $\left(p_{t}^{\phi}, y^{\phi}\right)$ bin. An example of invariant mass spectrum after background subtraction is presented in Fig. 5. The yield of the $\phi$ mesons is determined by fitting a relativistic Breit-Wigner distribution with parameters taken from the Particle Data Group compilation [28] (convoluted with the experimental resolution function obtained by a Monte Carlo simulation) superimposed on a linear background to account for a residual background in the low $p_{t}^{\phi}$ bins, to the measured line shape. The signal to background ratios vary from $1 / 2000$ to $1 / 180$ with increasing $p_{t}^{\phi}$. The signal is integrated in the mass range between $1.0 \mathrm{GeV} / \mathrm{c}^{2}$ and 
$1.05 \mathrm{GeV} / \mathrm{c}^{2}$. The resulting $\phi$ yields were corrected for acceptance and efficiency.

The efficiency- and acceptance-corrected $\phi$ meson yield is shown in Fig. 6 (left) for both decay modes as a function of transverse momentum. The inverse slope parameter of $T=273 \pm 9$ (stat) \pm 10 (sys) $\mathrm{MeV}$ and a rapidity density $d N / d y$ of $2.05 \pm 0.14$ (stat) \pm 0.25 (sys) in the $\mathrm{K}^{+} \mathrm{K}^{-}$decay mode and $T=306 \pm 82$ (stat) \pm 40 (syst) $\mathrm{MeV}$ and $d N / d y=2.04 \pm 0.49$ (stat) \pm 0.32 (syst) in the dilepton decay mode are in good agreement within the errors. A $\phi$ meson yield in the $\mathrm{e}^{+} \mathrm{e}^{-}$decay mode larger than 1.6 times the yield on the $\mathrm{K}^{+} \mathrm{K}^{-}$decay mode is excluded at $95 \% \mathrm{CL}$ (statistical and systematic errors in both decay channels added in quadrature).
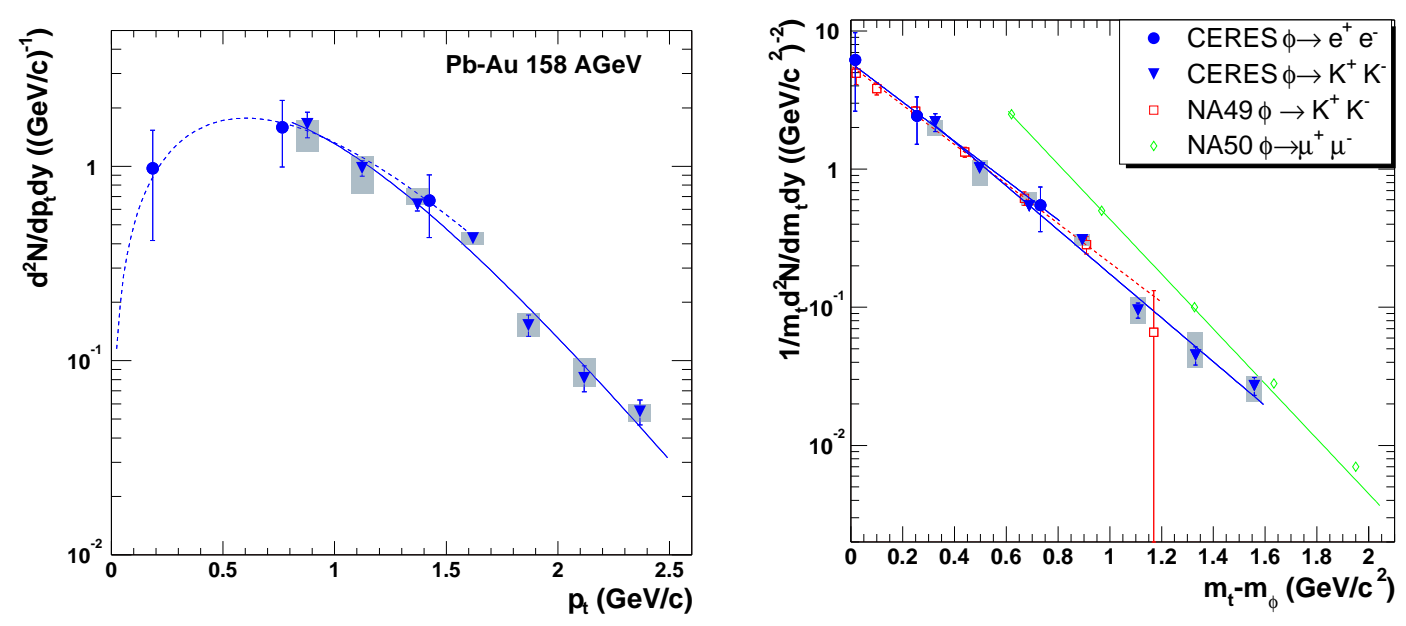

Figure 6: Left: Transverse momentum spectrum of $\phi$ mesons corrected for acceptance and efficiency reconstructed in the $\mathrm{e}^{+} \mathrm{e}^{-}$decay mode (circles) and in the $\mathrm{K}^{+} \mathrm{K}^{-}$decay channels (triangles). Systematic errors in the kaon decay channel are shown as boxes. The systematic errors in the dilepton analysis (not shown) are $\pm 16 \%$. Right: Transverse mass distribution of $\phi$ mesons measured in the charged kaon (triangles) and in the dilepton (circles) decay mode after scaling (see text) compared to the results from NA49 (squares) and NA50 (diamonds).

Moreover, the CERES results can be compared to the existing $\mathrm{Pb}-\mathrm{Pb}$ systematics [4] after accounting for the different measurement conditions. The NA49 measurement was done at $4 \%$ centrality and covered a rapidity range from 3 to 3.8 units [29]. A global scaling factor of $1.17 \pm$ 0.12 obtained experimentally is applied to the combined CERES data of Fig. 6 (left) to make the comparison to the systematics of [4]. In Fig. 6 (right) the scaled CERES $\phi$ transverse mass spectrum is plotted together with the NA49 and NA50 data. The $\phi$ meson yields agree within the errors with the NA49 results. So does the yield in the $\mathrm{K}^{+} \mathrm{K}^{-}$extrapolated down to $p_{t}=0$ using the measured inverse slope parameter. On the other hand, CERES data in the $\mathrm{K}^{+} \mathrm{K}^{-}$channel do not agree with NA50 results in the common $p_{t}$ region. This experiment however measures the leptonic channel. The extrapolation of NA50 results down to the region where CERES measures the dilepton channel does not agree either. As stated above, in the CERES measurement the two decay modes agree. Possible differences of maximum 40-50\% as expected by models including only rescattering of the kaons [3] or of maximum $70 \%$ at the lowest $p_{t}\left(p_{t}<0.3 \mathrm{GeV} / \mathrm{c}\right)$ expected by models including medium modifications of the $\phi$ mesons and kaons like the AMPT model [30] cannot be ruled out by the CERES results. 


\section{Conclusions}

To conclude, CERES has measured the low invariant mass spectrum of dilepton pairs with improved mass resolution. An enhancement of $2.56 \pm 0.22$ (stat) \pm 0.43 (syst) \pm 0.76 (decays) over the expectations of the hadronic decay cocktail is measured. The excess dilepton yield clearly favors models including a strong broadening of the $\rho$ spectral function and it rules out models including a mass shift of the $\rho$ meson.

At $40 \mathrm{AGeV}$, the observed pair yield is enhanced over the expectation from neutral meson decays by a factor of $5.9 \pm 1.5$ (stat) \pm 1.2 (syst) \pm 1.8 (decays). Compared to $158 \mathrm{AGeV}$ it is somewhat larger, with a significance of $1.8 \sigma$. The fact that the enhancement is somewhat larger and that the only quantity rising is the baryon density while the temperature decreases shows the importance of the baryon density for the in medium modifications of the $\rho$ meson.

Moreover, $\phi$ meson production has been measured simultaneously in both decay channels for the first time in relativistic heavy-ion collisions. The yield and the inverse slope parameter in both decay modes agree within the errors. Our results are in agreement with the results from NA49 measured in the kaon channel. A yield in the $\mathrm{e}^{+} \mathrm{e}^{-}$decay mode larger than 1.6 times the yield on the $\mathrm{K}^{+} \mathrm{K}^{-}$is excluded at $95 \% \mathrm{CL}$, therefore the large discrepancy observed previously is not observed in the CERES data. The theoretical predictions in $[3,30]$ are consistent with our data.

\section{Acknowledgments}

The CERES collaboration acknowledges the good performance of the CERN PS and SPS accelerators as well as the support from the EST division. We would like to thank R. Campagnolo, L. Musa, A. Przybyla, W. Seipp and B. Windelband for their contribution during construction and commissioning of the TPC and during data taking. We are grateful for excellent support by the CERN IT division for the central data recording and data proccesing. This work was supported by GSI, Darmstadt, the German BMBF, the German VH-VI 146, the US DoE, the Israeli Science Foundation, and the MINERVA Foundation.

\section{References}

[1] F. Karsch, J. Phys. G: Nucl. Part. Phys. 31 (2005) S633. F. Karsch, Nucl. Phys. A 698 (2002) 199c.

[2] V. Koch Int. J. Mod. Phys. E (1997) 203; F. Karsch, Nucl. Phys. A 590 (1995) 367c.

[3] S.C. Johnson, B.V. Jacak, A. Drees, Eur. Phys. J. C 18 (2001) 645.

[4] D. Röhrich, J. Phys. G 27 (2001) 355.

[5] G. Agakichiev et al. CERES Collaboration, Phys. Rev. Lett. 75 (1995) 1272.

[6] G. Agakichiev, CERES Collaboration, Eur. Phys. J. C 41, (2005) 475.

[7] G. Agakichiev et al. CERES Collaboration, Eur. Phys. Jour. C 4 (1998) 231.

[8] G.E. Brown and M. Rho., Phys. Rep. 363 (2002) 85.

[9] R. Rapp and J. Wambach, Adv. Nucl. Phys. 25 (2000) 1; private comunication.

[10] Addendum to proposal SPSLC/P280: CERN/SPSLC 96-35/P280 Add.1. 
[11] Technical Note on the NA45/CERES upgrade. CERN/SPSLC 96-50 (1996).

[12] A. Marín for the CERES Collaboration, Nucl. Phys. A 661 (1999) 673c.

[13] A. Marín et al., CERES Collaboration, J. Phys. G: Nucl. Part. Phys. 30 (2004) S709.

[14] D. Miśkowiec for the CERES Collaboration. Proc. Quark Matter 2005, Nucl. Phys. A 774 (2006) 43c.

[15] D. Adamova et al., CERES Collaboration, NIM in preparation.

[16] D. Adamova et al., CERES Collaboration, Phys. Rev. Lett. 91 (2003) 042301.

[17] C. Alt et al., NA49 Collaboration, Phys. Rev C 73(2006) 044910.

[18] D. Adamova et al., CERES Collaboration, submitted to Phys. Lett. B, nucl-ex/0611022v1

[19] D. Adamova et al., CERES Collaboration, Phys. Rev. Lett. 96 (2006) 152301.

[20] S. Yurevich, Doctoral Thesis, University of Heidelberg (2006).

[21] O. Busch, Doctoral Thesis, TU Darmstadt (2007).

[22] A. Cherlin, Doctoral Thesis, Weizmann Institute of Science (2005).

[23] H. Sako for the CERES Collaboration. Technical Report 03-25 (2000).

[24] R. Rapp, private comunication.

[25] K. Gallmeister et al., Nucl. Phys. A 688 (2001) 939; and B. Kämpfer, private communication.

[26] P. Braun-Munzinger, D. Miśkowiec, A. Drees, C. Lourenço, Eur. Phys. J. C 1 (1998) 123.

[27] J. Podolanski and R. Armenteros, Phil. Mag. 45 (1954) 13.

[28] S. Eidelmal et al., Phys. Lett. B 592 (2004) 1.

[29] S.V. Afanasiev et al., NA49 Collaboration, Phys. Lett. B 491 (2000) 59.

[30] S. Pal, C.M. Ko, Zi-wei Lin, Nucl. Phys. A 707 (2002) 525. 